Joanna Ciesielka

Università di Łódź

\title{
L'UNIONE SOVIETICA NEI MANIFESTI DELLA PROPAGANDA STALINISTA
}

\section{Soviet Propaganda Posters of the Stalinist Era}

\begin{abstract}
The visual culture represents relevant live evidence of a nation and of a country in the determined period of its development. The aim of my article is to analyse several Soviet propaganda posters of the Stalinist era created in the years 19241953 which provide an interesting image of the Soviet Union of the time. The analysed posters concern the issues such as war, enemies of communism, life of the nation and obviously, Stalin, a leader and dictator who was believed to hold URSS towards happiness and welfare. The subject of the analysis shall be both visual content of the posters and linguistic message conveyed by them.
\end{abstract}

Keywords: Soviet Union, Stalinist propaganda, propaganda posters, Stalinist era, linguistic analysis

\section{Riassunto}

La cultura visuale costituisce un'importante testimonianza della vita di una nazione o di un Paese in un determinato periodo del suo sviluppo. L'obiettivo del mio articolo è quello di analizzare dei manifesti di propaganda stalinista creati nell'URSS negli anni 1924-1953 che forniscono una interessante immagine dell'Unione Sovietica di quegli anni. I suddetti manifesti sottoposti ad analisi riguardano diversi temi, tra cui la guerra, i nemici del comunismo, la vita del popolo e, ovviamente, Stalin: il capo e il timoniere che guida l'Unione Sovietica verso la felicità e il benessere. Verrà analizzata sia la componente visuale dei manifesti che quella linguistica dei messaggi da essi veicolati.

Parole chiave: Unione Sovietica, propaganda stalinista, manifesto propagandistico, era stalinista, analisi linguistica 


\section{Introduzione}

La cultura visuale costituisce un'importante testimonianza della vita di una nazione o di un Paese. Essa viene anche usata dalle autorità nei sistemi totalitari nelle loro campagne propagandistiche; riportando le parole di Ramonet (Ramonet, 2000: 94), per «domare gli spiriti, violentare i cervelli e intossicare i cuori» ${ }^{1}$. Partendo dai film, passando per i dipinti e finendo con manifesti e slogan, il potere sovietico cercava di mobilitare il popolo per raggiungere gli scopi prefissati e orientare quest'ultimo verso il futuro (Volodina \& Alehin, 2015: 179). In questo articolo, una particolare attenzione verrà dedicata ai manifesti dato che - nel caso dell'Unione Sovietica - si può parlare di una produzione enorme di tale forma di comunicazione che per molti anni ha costituito una parte inseparabile della vita quotidiana dei cittadini di quello Stato. I manifesti erano presenti dappertutto: per le strade, nelle fabbriche, nelle scuole e negli uffici. Vi si trovavano risposte a diverse domande, contenevano suggerimenti su come vivere, definivano i confini tra il bene e il male. Come afferma G. P. Piretto (Piretto, 2003: 79):

(..) il manifesto, forma ridotta, sintetica ma fondamentale dell'operazione, che rilanciava concetti già noti o ne promuoveva di inediti, ma sempre sostenuto da parallele campagne che totalizzavano l'evento di cui era protagonista. Ultima tappa, non meno importante era la parola-immagine, i grandi slogan esibiti a caratteri cubitali su muri e tetti di edifici in ogni città, a riprendere il concetto chiave già introiettato dal pubblico attraverso più facili e godibili esperienze. Una sorta di ripasso costante, di memento in forma breve ma efficace di eventi visivi ed emotivi già vissuti e da non dimenticare.

Alla luce della quantità e del contenuto di queste opere grafiche si potrebbe affermare che nell'URSS il manifesto divenne un'arte «nazionale», che pur permettendo agli artisti di esprimersi in un certo modo, privilegiando il realismo espressivo, era sotto ispirazione e controllo statali.

L'obiettivo che mi pongo in questo testo è quello di analizzare alcuni manifesti di propaganda stalinista creati nell'URSS tra gli anni 1924-1953 e che veicolano un'interessante immagine dell'Unione Sovietica di quel periodo. Ho preso in considerazione cento tra le centinaia dei manifesti prodotti in quegli anni, suddividendoli in alcune categorie, quali: le

1 Traduzione propria. Segue la citazione in lingua originale: «domestiquer les esprits, violer les cerveaux et intoxiquer les cœurs». 
rappresentazioni di Stalin, l'esercito, la guerra, i nemici, il lavoro e lo sport. Dopo aver sottoposto ad un'analisi la componente visuale dei manifesti, procederò ad esaminarli dal punto di vista linguistico.

\section{Le categorie analizzate}

\subsection{Le rappresentazioni di Stalin}

L'ascesa al potere di Iosif Vissarionovic' Djugashvili detto Stalin (il soprannome proviene dalla parola stal' - acciaio) inizia subito dopo la morte di Lenin nel 1924ㄹ. Nel 1931 Stalin dichiarò l'infallibilità di Lenin chiamandolo vozhd' («capo» o «comandante»). In questo modo attribuì l'infallibilità anche a se stesso. La riflessione di quel modo di vedere sé, e il proprio predecessore ideologico, si osservano soprattutto nei manifesti creati negli anni 1928-1932. In questo periodo i profili dei due capi venivano raffigurati l'uno sullo sfondo dell'altro, proprio per sottolineare la continuazione dell'operato di Lenin da parte di Stalin. In quegli anni, Stalin veniva spesso rappresentato in una casacca militare, da solo, come timoniere. Nel manifesto di Deni e Dolgorukov del 19393, lo vediamo posto in posizione centrale con lo sguardo rivolto probabilmente verso il nemico e una mano in tasca, che costituisce una riproduzione di un gesto di Lenin. Inoltre, è da notare che la posa di Stalin nel manifesto sulla Piazza rossa non è casuale: proprio dietro di lui, quindi nascosto dalla sua figura, si trova il mausoleo di Lenin. La figura dello statista è enorme rispetto agli elementi che lo circondano: la sua figura domina non soltanto la Piazza ricoperta di soldati e di carri armati, ma anche il Cremlino che si trova alle sue spalle. Gli aerei sembrano degli uccelli che volano vicino ad un gigante. In questo manifesto, Stalin potrebbe essere paragonato alla Madre-Patria ${ }^{4}$ che accompagnerà i suoi soldati durante gli anni di lotta.

Negli anni Trenta e Quaranta, Stalin viene spesso rappresentato come bonario e sorridente, si potrebbe dire batiushka («caro e rispettato papà»), a cui il popolo si sottomette.

\footnotetext{
2 Per più informazioni si veda Bazylow, Wieczorkiewicz, 2005: 414-417.

3 https://warspot.ru/8408-berezhyom-stranu-rodnuyu (fot. 10) [15.03.2019].

4 Secondo lo storico russo G. P. Fedotov, nella religione popolare russa era presente «il costante desiderio di un grande potere divino femminile» (Hubbs, 1993: 3). L'archetipo femminile era associato alla Matushka Rus' «Madre Russia». La raffigurazione di Stalin che domina la Piazza Rossa fa pensare proprio a questo paradigma.
} 
È interessante, però, l'osservazione di G. P. Piretto il quale nota che spesso una barriera separa il capo dalla folla, ad esempio una tribuna. La sua figura non si mescola alla massa, è superiore ed unica. Si deve menzionare a questo riguardo l'opinione del suddetto studioso in base a cui, nella vita reale, Stalin non amava le situazioni in cui fosse attorniato dalla gente, proprio il contrario di ciò che veniva rappresentato in alcuni manifesti.

Dopo la guerra, i manifesti mostrano Stalin nei panni del gran maresciallo che ha combattuto la Germania nazista, in una postura solenne, con lo sguardo rivolto verso il futuro, con libri o bandiere rosse come sfondo. In un manifesto ${ }^{5}$ viene rappresentato con la pipa, elemento che gli dà un aspetto più rassicurante e umano.

\subsection{L’esercito: l'Armata Rossa}

Prima della seconda guerra mondiale, si proponevano manifesti che raffiguravano un esercito forte, come ad esempio quello del 19356: sulla Piazza Rossa si svolge una parata militare con i carri armati, la fanteria, il cielo viene solcato dagli aerei. Dietro le mura del Cremlino, sullo sfondo di una bandiera rossa, si vedono due figure gigantesche - una delle quali rappresenta Stalin e l'altra Kliment Efremovič Vorošilov, maresciallo dell'Unione Sovietica - che salutano l'Armata Rossa. A seguito dello scoppio della guerra, tornò il tema dell'estremo sacrificio (presente già prima nel discorso dell'inizio degli anni Venti) questa volta sotto la forma dello slogan: Za rodinu, za Stalina («Per la patria, per Stalin»). Da miglior esempio può servire il manifesto in cui si vede un soldato che, con la bandiera rossa in una mano e con il fucile nell'altra, corre verso il nemico per lottare per la patria e per Stalin, il suo condottiero. Finita la guerra numerosi manifesti raffiguravano soldati-vincitori, come si vede per esempio in uno, che rappresenta un soldato pluridecorato, con una stella rossa recante le insegne della falce e del martello; in un altro ${ }^{7}$ viene espressa la riconoscenza da parte del popolo nei confronti di un militare.

\subsection{La guerra}

Il tema della guerra coincide con quello dell'esercito. Per i sovietici, la guerra è iniziata solo il 22 giugno 1941 con l'invasione tedesca del territorio dell'URSS, ragion per cui nella storiografia russa viene chiamata «Velikaja

\footnotetext{
${ }^{5} \mathrm{http}: / /$ www.krasnoyeznamya.ru/pic.php? vrub=rm\&pid=10\&picid=88 [15.03.2019].

6 https://warspot.ru/8408-berezhyom-stranu-rodnuyu (foto 5) [15.03.2019].

7 https://artchive.ru/artists/9893 Plakaty_SSSR/works/280803 Ty_vernul_nam_zhizn' [15.03.2019].
} 
otchestvennaja vojna» (alla lettera: «la Grande guerra patriottica» ${ }^{8}$ ). Proprio nel 1941 apparve il manifesto La madre-patria chiama ${ }^{9}$ nel quale la Russia sovietica viene rappresentata come una donna vestita di rosso che tiene in una mano un foglio con il testo del giuramento di guerra e con l'altra fa il gesto di richiamare coloro che stanno dietro di lei, di cui si vedono solo fucili con le baionette inastate, segno di una lotta estrema, corpo a corpo.

Quel che sembra molto interessante è un altro manifesto, nei colori bianco, rosso e nero, in cui si raffigura un soldato dell'Armata Rossa che indica colui che guarda il poster chiedendogli: E tu cosa hai fatto per il fronte? ${ }^{10}$. La domanda del soldato sembra un grido, un'accusa, sullo sfondo di ciminiere fumanti e operai al lavoro, il che viene messo in rilievo dalla posizione e dalla scrittura in maiuscola della parola TU evidenziata in rosso sullo sfondo bianco e dal bianco dei caratteri del resto della frase che contrasta con lo sfondo nero. Vale la pena menzionare ancora un manifesto nella cui parte centrale è situato un grande carro armato nero recante la stella rossa sovietica, che, insieme da un secondo carro da cui è seguito, sta per schiacciare gli occupanti tedeschi tra cui Hitler, alcuni generali e soldati presi dal panico rappresentati in chiave ridicola. Dietro il carro armato sventola una bandiera rossa con la scritta: Avanti, all'Ovest!

\subsection{I nemici}

Gli anni 1937-1939 costituiscono il periodo del terrore staliniano detto anche «le grandi purghe». Le persone sospettate di essere cospiratori nel partito comunista, vengono fisicamente eliminate ${ }^{11}$. Appaiono le prime falsificazioni fotografiche: le persone indesiderabili, anche solo sospettate, vengono ritagliate via dalle foto e sostituite - ad esempio - da elementi architettonici, il che dimostra una crescente vena di paranoia di Stalin. Nei manifesti dell'epoca viene riflessa anche la paura delle spie, vale a dire, tra gli altri, degli agenti segreti stranieri. Uno dei poster più noti che illustra questa tendenza è del 1941 e rappresenta una donna con un fazzoletto rosso sulla testa che si mette un indice sulle labbra, in un gesto che intima il silenzio. La scritta Ne boltaj («Non chiacchierare») ${ }^{12}$ completa l'immagine.

\footnotetext{
8 Nella storiografia italiana si usa il termine «Fronte Orientale».

9 https://gallerix.ru/storeroom/1973977528/N/1019548323/ [15.03.2019].

$10 \mathrm{https} / /$ gallerix.ru/storeroom/1973977528/N/1000920762/ [15.03.2019].

11 Per saperne di più, si veda Bazylow, Wieczorkiewicz, 2005: 435-444.

12 https://gallerix.ru/storeroom/1973977528/N/1732069683/ [15.03.2019].
} 
Un altro esempio che volevo riportare, benché ideato un anno dopo la morte di Stalin, nel 1954, da V. Koretskij ${ }^{13}$, è degno di nota dal punto di vista della rappresentazione del nemico: la figura è divisa in due parti, l'agente (probabilmente uno straniero) e un individuo invece dalla fisionomia familiare, conosciuta. Il messaggio è il seguente: attenzione a chi dai informazioni, perché dietro ad ogni conoscente può nascondersi una spia al servizio del nemico. È interessante il gesto del «chiacchierone», cioè del pollice rivolto dietro di sé, in quanto fa capire che non parla di sé ma di qualcun altro o comunque di una situazione che non lo riguarda direttamente.

\subsection{Il lavoro}

Soffermandoci sul tema del lavoro, non si può evitare di citare le parole-chiave dei tempi dell'Unione Sovietica: industrializzazione e collettivizzazione. Quest'ultima si riferisce alla istituzione delle fattorie collettive kolchoz. Il primo manifesto in proposito che vorrei descrivere rappresenta due donne immerse in un campo di grano: la prima, sulla sinistra, appare come un membro dell'apparato sovietico, reca ben due medaglie sul petto; è tenuta a braccetto da una contadina con una medaglia (può darsi che si tratti del riconoscimento dato ai grandi lavoratori).

L'immagine è del tutto positiva: il giorno è bello, assolato, il cielo azzurro. Le protagoniste sorridono immerse tra spighe di grano già alte e rigogliose; ciò rappresenta un segno evidente di un raccolto abbondante che lascia intravedere all'orizzonte un futuro altrettanto rigoglioso. Le due protagoniste sembrano felici. Questa immagine idilliaca viene accompagnata da una citazione di Stalin: Le donne nei kolchoz: una grande forza. In un altro manifesto si vedono due giovani sorridenti, una ragazza e un ragazzo, quest'ultimo probabilmente è alla guida di un trattore. Stanno chiamando qualcuno dicendo, senza aria di accusa ma con simpatia: Compagno, vieni da noi nella fattoria collettiva ${ }^{14}$ !

Per presentare altri manifesti legati al lavoro non si può fare a meno di nominare Stachanov, un minatore che nel 1935 riuscì a battere un record di produzione (Piretto, 2018: 191). Il suo nome dà origine alla nozione «stacanovismo» il fenomeno il cui obiettivo era quello di aumentare la produttività.

13 http://www.krasnoyeznamya.ru/pic.php?vrub=rm\&pid=10\&picid=874 [15.03.2019].

14 https:/gallerix.ru/storeroom/1973977528/N/934354374/ [15.03.2019]. 
All'aumentare dei toni trionfalistici, con cui viene celebrato il lavoro, corrisponde la spinta verso l'iperproduttività. Gli stacanovisti sono premiati: a loro spettano biciclette, grammofoni, vita agiata e comoda. Si progettano dei piani quinquennali che si cerca di realizzare in un periodo più breve.

Un manifesto che illustra tale situazione è quello in cui è rappresentato un'operaia, probabilmente una saldatrice, su un'impalcatura, che rivolge una domanda a chi guarda il poster: Noi abbiamo raggiunto la quota. E voi? Nella stessa «corrente» si iscrive il manifesto che raffigura un muratore in primo piano accompagnato dalle sue parole: Costruire bene, velocemente e a buon mercato ${ }^{15}$.

\subsection{Lo sport}

Lo sport era un altro aspetto importante della vita dell'Unione Sovietica. Soprattutto dopo la guerra si incitavano i giovani a praticare diverse discipline, non perché rimanessero in forma, ma nello scopo di battere nuovi record e contribuire in questo modo alla grandezza del Paese. In questa sede riportiamo due esempi di manifesti legati allo sport. Nel primo, allo stadio, viene rappresentata una donna che sta per lanciare il giavellotto. Nel secondo, un uomo intento al lancio del peso. Le scritte che accompagnano le immagini confermano quanto è stato scritto sopra: Giovani! Agli stadi! e Sportivi, lottate per stabilire nuovi record ${ }^{16}$.

\section{Analisi linguistica}

Come afferma G. Durandin (Durandin, 1983: 23): «l'obiettivo della propaganda è quello di esercitare un'influenza sull'individuo o su un gruppo sia per farlo agire in un senso dato sia per renderlo passivo e dissuaderlo da opporsi a certe azioni» ${ }^{17}$.

I manifesti svolgono questa funzione non solo attraverso l'immagine, ma anche attraverso il testo che ne fa parte. L'idea di poter agire sulla gente usando il linguaggio è conosciuta sin dall'Antichità, in questa sede, visto lo spazio a disposizione, non è stato possibile fare una rassegna di teorie proposte al riguardo. Mi riferirò solo alla teoria degli atti linguistici

15 http://www.fishki.lv/index.php?newsid=24269 [15.03.2019].

16 http://www.savok.org/plakaty/543-plakaty.html [15.03.2019].

17 Traduzione propria. «La propagande a pour but d'exercer une influence sur l'individu ou sur un groupe soit pour le faire agir dans un sens donné soit pour le rendre passif et le dissuader de s'opposer à certaines actions». 
di Austin e Searle, i maggiori ideatori della teoria pragmatica della comunicazione della linguistica contemporanea.

Gli atti linguistici presenti nei testi dei manifesti si possono dividere in quattro gruppi: assertivi, direttivi, commissivi ed espressivi. Ognuno di essi verrà descritto con gli appositi esempi.

\subsection{Atti assertivi}

Gli atti assertivi si evidenziano nei messaggi riguardanti la situazione nell'Unione Sovietica e il suo capo (ovviamente quelli ai quali l'apparato della propaganda ci vuole far credere). Tra gli esempi possiamo riportare:

Капитан страны Советов ведет нас от победы к победе! [Il capitano dell'Unione Sovietica ci guida di vittoria in vittoria!];

Любимый Сталин - счастье народное! [L'amato Stalin è la felicità del popolo!] 18;

Великий Сталин - светоч коммунизма! [Il grande Stalin - fiaccola del comunismo!];

Сталинским духом крепка и сильна армия наша и наша страна! [Forti e saldi dello spirito staliniano sono il nostro esercito e il nostro paese!];

Женщины в колхозах - большая сила. (И. Сталин) [Le donne nei kolchoz: una grande forza. (I. Stalin)] ${ }^{19}$.

Nelle frasi testè citate possiamo osservare due modi in cui si adopera un riferimento a Stalin: in alcuni casi viene nominata direttamente la sua persona (nelle parole: Stalin, capitano dell'Unione Sovietica), in altri, egli viene indirettamente evocato (attraverso l'espressione spirito staliniano, che caratterizza l'esercito e l'Unione Sovietica). Si nota anche l'uso di aggettivi qualificativi: da una parte quelli relativi alla persona del vozh'd, cioè любимый е великий («amato» е «grande») e dall'altra quelli caratterizzanti l'URSS e l'Armata Rossa: крепка и сильна («forte» е «salda»). I due primi potrebbero essere interpretati come segni dell'instaurazione del culto della personalità tipico dei paesi del sistema totalitario: il capo viene descritto con l'epiteto di «grande», che ha un significato molto generico e permette di

\footnotetext{
18 http://www.sovposters.ru/view/177/ [15.03.2019].

19 https:/gallerix.ru/storeroom/1973977528/N/1675214687/ [15.03.2019].
} 
attribuire alla persona un vasto numero di tratti positivi; la parola «amato » si riferisce invece alle emozioni provate dagli abitanti dell'Unione Sovietica nei confronti della figura del comandante, elemento che viene maggiormente messo in rilievo dal predicato della frase: «è la felicità del popolo».

Vale la pena menzionare l'uso degli aggettivi possessivi e dei pronomi. La «bella realtà», rappresentata negli atti assertivi, è relativa all'Unione Sovietica «sotto la guida di Stalin», per riprendere le parole di uno degli slogan. Lo indicano le forme quali: нас «noi» e наша «la nostra» (che, nella traduzione dell'esempio sopra citato, figura come il «nostro»).

\subsection{Atti direttivi}

Negli atti direttivi l'obiettivo del locutore è quello di convincere l'interlocutore a compiere un'azione oppure dissuaderlo da essa. Osserviamo alcuni esempi:

Иди, товарищ, к нам в колхоз! [Compagno, vieni da noi nella fattoria collettiva!];

Не болтай! [Non chiacchierare!] ${ }^{20}$;

Под знаменем Ленина, под водительством Сталина - вперед, к победе коммунизма! [Sotto l'insegna di Lenin, sotto la guida di Stalin, avanti verso la vittoria del comunismo!] $]^{21}$;

Под водительством великого Сталина - вперед, к коммунизму! [Sotto la guida del grande Stalin, avanti verso il comunismo!];

Молодежь - на стадионь! [Giovani! Agli stadi!];

Все мировые рекорды должны быть нашими! [Tutti i record del mondo dovrebbero essere nostri!];

Mы выполнили норму... a вы ? [Noi abbiamo raggiunto la quota. E voi?].

Nei primi due casi l'esortazione e l'ordine vengono espressi con la seconda persona singolare dell'imperativo. È da notare l'uso della parola товарищ «compagno», caratteristico per il linguaggio dei soviet. I due seguenti esempi assumono la forma di slogan esortativi verso la vittoria del comunismo.

20 https:/gallerix.ru/storeroom/1973977528/N/1732069683/ [15.03.2019].

21 http://www.sovposters.ru/view/155/ [15.03.2019]. 
Tra i messaggi veicolati dagli atti direttivi si riscontrano anche quelli realizzati con le proposizioni ellittiche: nel caso di Молодежь - на стадионы! il verbo è rimasto sottointeso.

Un caso interessante costituisce lo slogan che vede implicato l'uso del verbo быть должным («dovere» al condizionale), nel quale l'esortazione rimane indiretta. L'ultimo atto direttivo citato non termina, caso unico tra tutti gli esempi, con un punto esclamativo, ma con un'interrogazione. La constatazione compresa nella prima parte del messaggio può essere intesa come l'affermazione dei comunisti veri, la quale, però, seguita dalla domanda $a b b l$ «E voi?» costituisce un invito alla riflessione, competizione, e anche a seguire l'esempio dei «bravi compagni».

\subsection{Atti commissivi}

Gli atti commissivi consistono in una promessa o in una minaccia da parte del locutore. Nei manifesti analizzati sono state evidenziate le seguenti scritte appartenenti a questa categoria:

Выполним план великих работ! [Porteremo a compimento il piano delle grandi opere! ]22;

Придем к изобилию! [Raggiungeremo l'abbondanza!]23.

Ambedue le promesse sono state espresse in modo diretto, con la prima persona plurale del futuro. Quanto all'aspetto linguistico, risulta che la forma grammaticale usata si riferisce a Stalin e al popolo dell'Unione Sovietica che gli terrà dietro.

\subsection{Atti espressivi}

L'intenzione comunicativa che costituisce la base degli atti espressivi è quella di ringraziare o salutare l'interlocutore, oppure di rivolgergli un qualche augurio. Tra gli esempi riscontrati, le forme seguenti sono degne di una particolare attenzione:

Слава воину - победителю! [Gloria al soldato vincitore!];

Смерть немецким оккупантам! [Morte agli invasori tedeschi!];

\footnotetext{
22 https://gallerix.ru/storeroom/1973977528/N/2029492133/ [15.03.2019].

23 http://www.sovposters.ru/view/161/ [15.03.2019].
} 
Да здравствует рабоче-крестьянская Красная Армия - верный страж советских грании!! [Lunga vita all'Armata Rossa operaio-contadina, la fedele guardia dei confini sovietici!];

Ты вернул нам жизнь! [Ci hai restituito la vita!];

Спасибо любимому Сталину - за счастливое детство! [Grazie all'amato Stalin per la nostra infanzia felice!];

Советские физкультурники - гордость нашей страны [Atleti sovietici - orgoglio del nostro Paese].

I due primi dai tre messaggi-slogan che esprimono un augurio (Слава..., Смерть..., Да здравствует...) hanno una forma nominalizzata senza predicato. La lode e i buoni auguri riguardano l'esercito operaiocontadino fiero di aver vigilato i confini del Paese e di aver vinto la Grande Guerra Patriottica, d'altro canto l'augurio di morte si indirizza al nemico tedesco. Il quarto messaggio è stato espresso con la frase comprendente solo il verbo вернуть al passato, per cui non sembra appartenere agli atti espressivi. Nonostante ciò, potrebbe anche essere trattato come uno di essi in quanto l'intenzione del locutore (e ciò emerge chiaramente nella componente visuale del manifesto) è senz'altro quella di ringraziare. Stalin viene ringraziato, in modo diretto, con la parola спасибо. È da notare l'uso dell'aggettivo qualificativo любимый «amato» davanti al nome del leader sovietico. L'ultimo messaggio riportato esprime orgoglio. Dal punto di vista formale la proposizione è ellittica, senza predicato.

\section{Conclusione}

Qual è l'immagine dell'Unione Sovietica che emerge dai manifesti propagandistici del periodo analizzato? Quella di un Paese molto potente, con sovrastanti forze armate pronte a difendere i propri confini dall'attacco di ogni nemico, un mondo guidato da un comandante forte e visionario, impegnato a proseguire la strada del suo grande predecessore, quella del comunismo. Un Paese il cui condottiero viene dal popolo ed è a lui vicino, un condottiero che, come un padre, chiuso all'interno delle mura del Cremlino, si preoccupa di ognuno dei suoi figli. Emerge quindi la figura di un capo-stratega geniale grazie al quale la Germania nazista è stata sconfitta. Nei manifesti si vede l'Unione Sovietica dei giovani operai 
e kolchozniki, tutti impegnati a lavorare con ardore nelle fabbriche e nelle fattorie collettive per portare a termine con successo i piani quinquennali, l'Unione Sovietica degli stacanovisti e degli sportivi - che superando con zelo i limiti delle proprie possibilità contribuiscono alla creazione di una società socialista. Una società che però è minacciata da insidiosi nemici (che possono nascondersi dappertutto) dai quali ci si deve guardare, che sono invidiosi dei progressi dello sviluppo socialista e della felicità che è riuscita a raggiungere.

Guardando i manifesti sovietici, non solo quelli del periodo sottoposto ad analisi, sarebbe giusto evocare la nozione di iperrealtà, di un costante gioco tra il presente e il futuro, il che si manifesta attraverso la presentazione della vita quale sarà, e non qual è. Lo si può osservare anche nelle pubblicità dell'epoca (ad esempio in una di esse si parla dei mantelli di scoiattolo: prodotto reclamizzato ancor prima che fosse disponibile sul mercato).

Il linguaggio usato nei manifesti è semplice. Dal punto di vista lessicale e morfosintattico si possono distinguere in esso alcuni tratti caratteristici, vale a dire: l'uso di parole ed espressioni quali compagno, piano (da realizzare), kolchoz, raggiungere la quota, sovietico, operaio-contadino, comunismo; l'uso degli aggettivi, come grande, amato davanti al nome di Stalin; l'opposizione noi vs. loro (nemici); la presenza di numerose frasi esclamative; l'uso delle frasi ellittiche e degli slogan. Nei manifesti sottoposti all'analisi dominano gli atti assertivi e direttivi.

\section{Bibliografia}

Bazylow, L. \& Wieczorkiewicz, P. (2005). Historia Rosji, t. 2. Wrocław: Ossolineum. Charaudeau, P. (2009). Le discours de manipulation entre persuasion et influence sociale. Actes du colloque de Lyon, http://www.patrick-charaudeau.com/Lediscours-de-manipulation-entre.html [10.02.2019].

Durandin, G. (1983). L'information, la désinformation et la réalité. Paris: PUF.

Hubbs, J. (1993). Mother Russia: The Feminine Myth in Russian Culture. Bloomington: Indiana University Press.

Piretto, G. P. (2003). Visioni e rappresentazioni di un non-flâneur sovietico: lo sguardo del e sul compagno Stalin. Culture, Annali del Dipartimento di Lingue e Culture Contemporanee della Facoltà di Scienze Politiche dell'Università degli Studi di Milano, 17, pp. 71-86, http://www.club.it/culture/culture2003/ gian.piero.piretto/corpo.tx.piretto.html [17.01.2019].

Piretto, G. P. (2018). Quando c'era l'URSS. 70 anni di storia culturale sovietica. Milano: Raffaello Cortina Editore. 
Ramonet, I. (2000). Propagandes silencieuses. Masses, télévision, cinéma. Paris: Galilée. Володина, Н. А. \& Алехин, Э. В. (2015). Советский плакат в системе большевистской пропаганды:образ будущего (1917-1941 гг.). Теория и практика общественного развития, № 1 (№21), pp. 179-181, http:// teoria-practica.ru/rus/files/arhiv_zhurnala/2015/20/sociology/volodinaalekhin.pdf [17.01.2019]. 